\title{
Diagnostic Factors of Odontogenic Cysts in Iranian Population: A Retrospective Study Over the Past Two Decades
}

\author{
Hassan Mohajerani ${ }^{1}$; Mohammad Esmaeelinejad ${ }^{1, *}$; Siamak Sabour ${ }^{2}$; Farzad Aghdashi ${ }^{1}$; \\ Nima Dehghani ${ }^{1}$ \\ ${ }^{1}$ Department of Oral and Maxillofacial Surgery, School of Dentistry, Shahid Beheshti University of Medical Sciences, Tehran, IR Iran \\ ${ }^{2}$ Department of Clinical Epidemiology, School of Dentistry, Shahid Beheshti University of Medical Sciences, Tehran,IR Iran \\ ${ }^{*}$ Corresponding Author: Mohammad Esmaeelinejad, Department of Oral and Maxillofacial Surgery, School of Dentistry, Shahid Beheshti University of Medical Sciences, Tehran, \\ IR Iran. Tel: +98-2166353674, Fax: +98-2122439976, E-mail: esmaeelnejad@gmail.com
}

Received: July 19, 2014; Revised: September 10, 2014; Accepted: September 23, 2014

\begin{abstract}
Background: Early diagnosis of odontogenic cysts due to their silent progression is always a challenging problem for clinicians. Objectives: The current study aimed to evaluate the frequency of odontogenic cysts and related factors in a selected Iranian population. Patients and Methods: The current cross-sectional study was conducted on 312 patients' recorded data in Taleghani Hospital, Tehran, Iran, from April 1993 to December 2013. All related data were extracted from the records and categorized in tables. The correlation between the variables was analyzed by either chi-square or multinominal logistic regression tests. The P values $<0.05$ were considered significant. Results: Evaluation of 312 patients' records (185 males and 127 females) with the mean age of 27.6 showed that Odontogenic Keratocyst (OKC) was the most common odontogenic cyst of all followed by the dentigerous cyst as the second most common lesion. Most of the patients were in the second or third decades of their lives, although there was no statistically significant age distribution. The finding of the current study showed that calcifying odontogenic cyst(COC)occurrence was significantly related to the history of trauma. Enucleation and curettage of the odontogenic cysts were the most common treatment plans of all.

Conclusions: The current study showed that clinicians should consider the many factors associated with the occurrence of odontogenic cysts.
\end{abstract}

Keywords: Epidemiology; Etiology; Odontogenic Cyst; Odontogenic Keratocyst

\section{Background}

Odontogenic cysts are one of the most common pathologic lesions in maxillofacial region. Odontogenic cysts arise from proliferation of the odontogenic cell rests such as the epithelial cells of Malassez and cell rests of Serres in jaws. Odontogenic cysts are categorized into two groups of inflammatory and developmental cysts based on the pathologic process of occurrence (1-3). These pathologic lesions are slow-growing and noninvasive in most cases. Odontogenic cysts are benign pathologic lesions; however they may cause some serious problems such as tooth mobility or jaw fracture. These important complications can be due to the late diagnosis of cysts because of their slow and stealthy progression $(4,5)$.

The prevalence of these lesions is different in various populations and is reported in several investigations (6-8). The frequency of different types of odontogenic cysts and their associated factors are important to manage the cysts and employ an appropriate treatment plan. Several researches suggested the different factors affecting the incidence of odontogenic cysts. Age, gender, and trauma are some of these associated agents observed in relation to odontogenic cysts (9-11). Identifying the target age group in different cysts and radiographic presentation of these lesions are important in their early detection and treatment. In several articles the prevalence of various odontogenic cysts in different age groups and genders are reported (11-13). The radiographic views of odontogenic cysts are evaluated in some other investigations $(14,15)$.

Some investigations reported the prevalence of such cysts in Iranian population, although they were local studies and there is no consensus on the definite effects and associated factors in the developing odontogenic cysts (16-18).

\section{Objectives}

Since early diagnosis of odontogenic cysts simplifies the treatment, and the prevalence of odontogenic cysts is different in various populations, the current study aimed to evaluate the factors affecting odontogenic cysts and their prevalence in the Iranian population and compare the findings with those of the previous studies. 


\section{Patients and Methods}

The current retrospective study was carried out on the patients' records referred to the Department of Oral and Maxillofacial Surgery, Ayatollah Taleghani Hospital, Tehran, Iran in the past two decades (1993 - 2013). The data from the patients' records including clinical examination charts, histopathology records, and imaging records were collected in a categorized chart. In each case the following information was obtained:

- Age

- Gender

- Presence of pain, hypoesthesia, anesthesia, and paresthesia

- History of trauma

- Presence of infection

- Positivity of aspiration

- Simultaneous with impacted tooth

- The locations of odontogenic cysts were divided into anterior (distal surface of canine on one side) and posterior (distal to canines) for each jaw.

- The treatment approaches were divided into categories of enucleation; enucleation and curettage; marsupialization; marsupialization followed by enucleation; marsupialization followed by enucleation and curettage.

- The radiographic patterns of cysts were divided into four categories: unilocular cysts with well-defined borders; unilocular cysts with ill-defined borders; multilocular cysts with well-defined borders; multilocular cysts with ill-defined borders.

All data were extracted by an expert oral and maxillofacial surgeon.

The inclusion criteria for subject selection were:

1- Hospitalization between April 1993 and December 2013

2- Referring for the first time

3- Complete records with no missing variable

4-A confirmed odontogenic cyst report

5- Available panoramic view of the cyst

The following subjects were excluded from the study:

1- Referring to the hospital before 1993 or after 2013

2- Hospitalized more than once

3- Records with some missing data

4- Unclear pathologic report

The last classification of odontogenic cysts and tumors published by the World Health Organization (WHO) in 2005 was used to categorize the cysts extracted from the pathologic reports. The Odontogenic Keratocyst (OKC) was considered as a cystic lesion in the current study; however it was defined as a benign tumor and classified as a keratocystic odontogenic tumor.

In the second part of the study a systematic review of the articles investigating the prevalence of odontogenic cysts in Iranian population was implemented. There were three articles in the English literature regarding the Iranian population to reveal a demographic distribution of odontogenic cysts. All of the above mentioned variables were extracted from these articles and categorized in a table.

\subsection{Ethical Considerations}

The current study was designed according to the hospital's ethics committee rules. The name and personal identifications of the patients were omitted from the study and not mentioned.

\subsection{Statistical Analysis}

Sample size calculation was performed by PASS software (version 12.0.8; NCSS, LLC, Utah, USA) considering $\alpha=$ 0.05 and power of 0.9. The estimated sample size was 254 subjects. In order to design a comprehensive study the records of the past twenty years were evaluated in which 312 subjects were included according to the inclusion criteria. As a matter of fact the subjects were selected as an available sampling strategy.

All calculations were processed using Statistical Package for Social Science statistical software (version 20; SPSS Inc.). Descriptive statistics including tables and graphs were applied to show the information. Chi-square test was used to determine the significant differences between the frequency of odontogenic cysts and other variables. A P value $<0.05$ was considered statistically significant.

In multinominal logistic regression, considering the OKC as the reference group, the relationships between trauma, location of the cyst, impacted tooth, age, gender, and motor and sensory nerve functions were assessed. AP value $<0.05$ was considered statistically significant.

\section{Results}

Records of all the subjects with diagnosis of pathologic lesion were assessed in the current study. The total pathology reports available in the hospital were 3875 cases, of which 3466 had referred to the hospital for the first time. Three hundred and forty eight cases were cysts of the jaw, among which 312 were odontogenic cysts ( $9 \%$ of all pathologic lesions) and were included in the study according to the inclusion criteria. The subjects' age varied from 9 to 77 years with the mean age of $27.6 \pm 13.47$ years. The relationship between age and the pathologic lesion was statistically significant $(P=0.05)$. Most of the subjects with OKC diagnosis were in the third and second decades of their liver (36\% and $28 \%$ respectively) whilst patients with dentigerous cysts were mostly in the second decade (42.5\%) (Figure 1). One hundred and eighty five patients were male (59.2\%) and 127 cases (40.7\%) were female (male to female ratio 1.45). Although the frequency of the male subjects was more than that of female cases the difference was not statistically significant $(P=0.41) .29 .2 \%$ of the cases were inflammatory cysts while $70.8 \%$ of them were developmental cysts (91 versus 221 cases). Odontogenic keratocyst (OKC) and dentigerous cysts were the most common cysts $(32.1 \%$ and $25.6 \%$ respectively) (Figure 2 ). History of trauma was not related to most of the cases $(\mathrm{P}=0.001)$. There was no 
relationship between pain or sensory nerve dysfunction or infection and the pathologic lesions ( $P$ values were $0.06,0.28$, and 0.57 , respectively). The third molar was the most common impacted tooth in OKC and detigerous cysts (34\% and $47.5 \%$ of the cases respectively). These findings were statistically significant ( $P$ $=0.001)$. The most common location of OKC and dentigerous cysts was the posterior of the mandible ( $\mathrm{P}=$ $0.002)$. The findings regarding the location of the cysts are shown in Table 1 . The amber colored fluid was significantly more common (28.6\% of the cases) in aspiration of odontogenic keratocysts $(P=0.01)$. there was a statistically significant relationship In multinominal logistic regression, considering the OKC as the reference group, to assess the relationship between trauma, location of the cyst, impacted tooth, age, gender and sensory nerve dysfunction, between calcifying odontogenic cyst $(\mathrm{COC})$ and trauma $\mathrm{OR}=1.2 ; \mathrm{P}=0.015$, impacted tooth and dentigerous cyst $\mathrm{OR}=2.1 ; \mathrm{P}=0.044$ (Table 2).

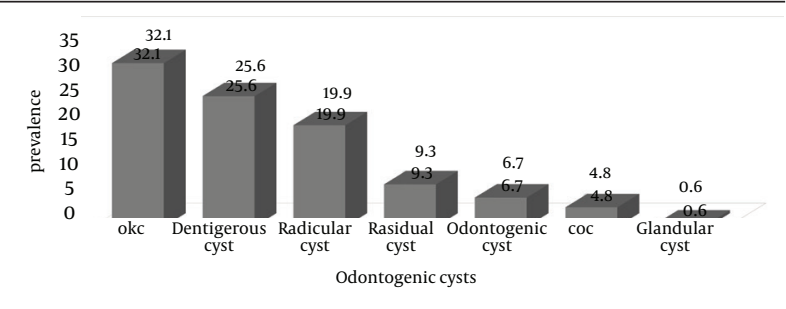

Figure 1. Prevalence of Different Odontogenic Cysts in the Population

Figure 2. Age Distribution of Odontogenic Cysts in the Population

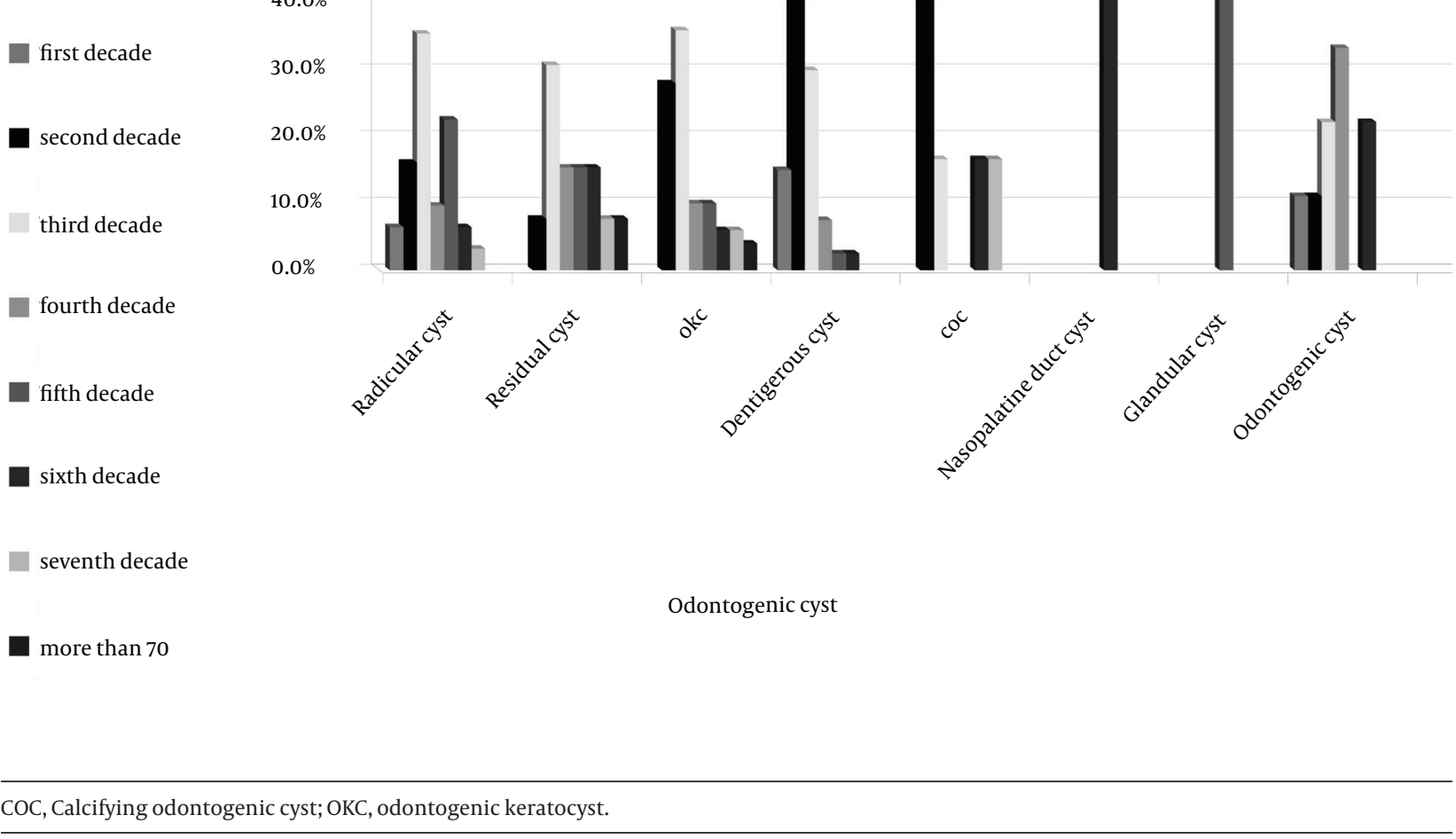


The elective treatment plan for each pathologic lesion is shown in Figure 3.

In the second part of the study the extracted data from three previous published articles and the findings of the current study are indicated in Table 3. These four studies showed that during the past twenty five years in different locations of Iran odontogenic cysts were more common in men with high prevalence of radicular cyst.

Table 1. The Location of Different Odontogenic Cysts in Human Jaws a

\begin{tabular}{|c|c|c|c|c|c|c|c|c|c|c|c|c|c|c|c|c|}
\hline \multirow[t]{2}{*}{$\begin{array}{l}\text { Location of The } \\
\text { Cyst in Jaw }\end{array}$} & \multicolumn{2}{|c|}{$\begin{array}{c}\text { Radicular } \\
\text { Cyst }\end{array}$} & \multicolumn{2}{|c|}{$\begin{array}{l}\text { Residual } \\
\text { Cyst }\end{array}$} & \multicolumn{2}{|c|}{ OKC } & \multicolumn{2}{|c|}{$\begin{array}{c}\text { Dentigerous } \\
\text { Cyst }\end{array}$} & \multicolumn{2}{|c|}{$\begin{array}{c}\text { Nasopalatine } \\
\text { Duct Cyst }\end{array}$} & \multicolumn{2}{|c|}{$\begin{array}{c}\text { Glandular } \\
\text { Cyst }\end{array}$} & \multicolumn{2}{|c|}{$\mathrm{COC}$} & \multicolumn{2}{|c|}{$\begin{array}{c}\text { Odontogenic } \\
\text { Cyst }\end{array}$} \\
\hline & $\mathrm{N}$ & $\mathrm{P} \%$ & $\mathrm{~N}$ & $\mathrm{P} \%$ & $\mathrm{~N}$ & $\mathrm{P} \%$ & $\mathrm{~N}$ & $\mathrm{P} \%$ & $\mathrm{~N}$ & $\mathrm{P} \%$ & $\mathrm{~N}$ & $\mathrm{P} \%$ & $\mathrm{~N}$ & $\mathrm{P} \%$ & $\mathrm{~N}$ & $\mathrm{P} \%$ \\
\hline $\begin{array}{l}\text { Right maxillary } \\
\text { posterior region }\end{array}$ & 1 & 16.1 & 3 & 23.1 & 6 & 12.0 & 5 & 12.5 & 0 & 0 & 1 & 100 & 1 & 16.7 & 0 & 0 \\
\hline $\begin{array}{l}\text { Anterior maxillary } \\
\text { region }\end{array}$ & 7 & 22.6 & 2 & 15.4 & 1 & 2.0 & 6 & 15.0 & 2 & 100 & 0 & 0 & 2 & 33.3 & 1 & 11.1 \\
\hline $\begin{array}{l}\text { Left maxillary } \\
\text { posterior region }\end{array}$ & 3 & 9.7 & 3 & 23.1 & 3 & 6.0 & 1 & 2.5 & 0 & 0 & 0 & 0 & 0 & 0 & 1 & 11.1 \\
\hline $\begin{array}{l}\text { Right mandibular } \\
\text { posterior region }\end{array}$ & 5 & 16.1 & 0 & 0 & 17 & 34.0 & 11 & 27.5 & 0 & 0 & 0 & 0 & 2 & 33.3 & 3 & 33.3 \\
\hline $\begin{array}{l}\text { Anterior mandibu- } \\
\text { lar region }\end{array}$ & 4 & 12.9 & 1 & 7.7 & 5 & 10.0 & 6 & 15.0 & 0 & 0 & 0 & 0 & 0 & 0 & 0 & 0 \\
\hline $\begin{array}{l}\text { Left mandibular } \\
\text { posterior region }\end{array}$ & 7 & 22.6 & 4 & 30.8 & 18 & 36.0 & 11 & 27.5 & 0 & 0 & 0 & 0 & 1 & 16.7 & 4 & 44.4 \\
\hline
\end{tabular}

a Abbreviations: COC, calcifying odontogenic cyst; and OKC, odontogenic keratocyst.

Figure 3. Treatment Plans for Odontogenic Cysts in the Population

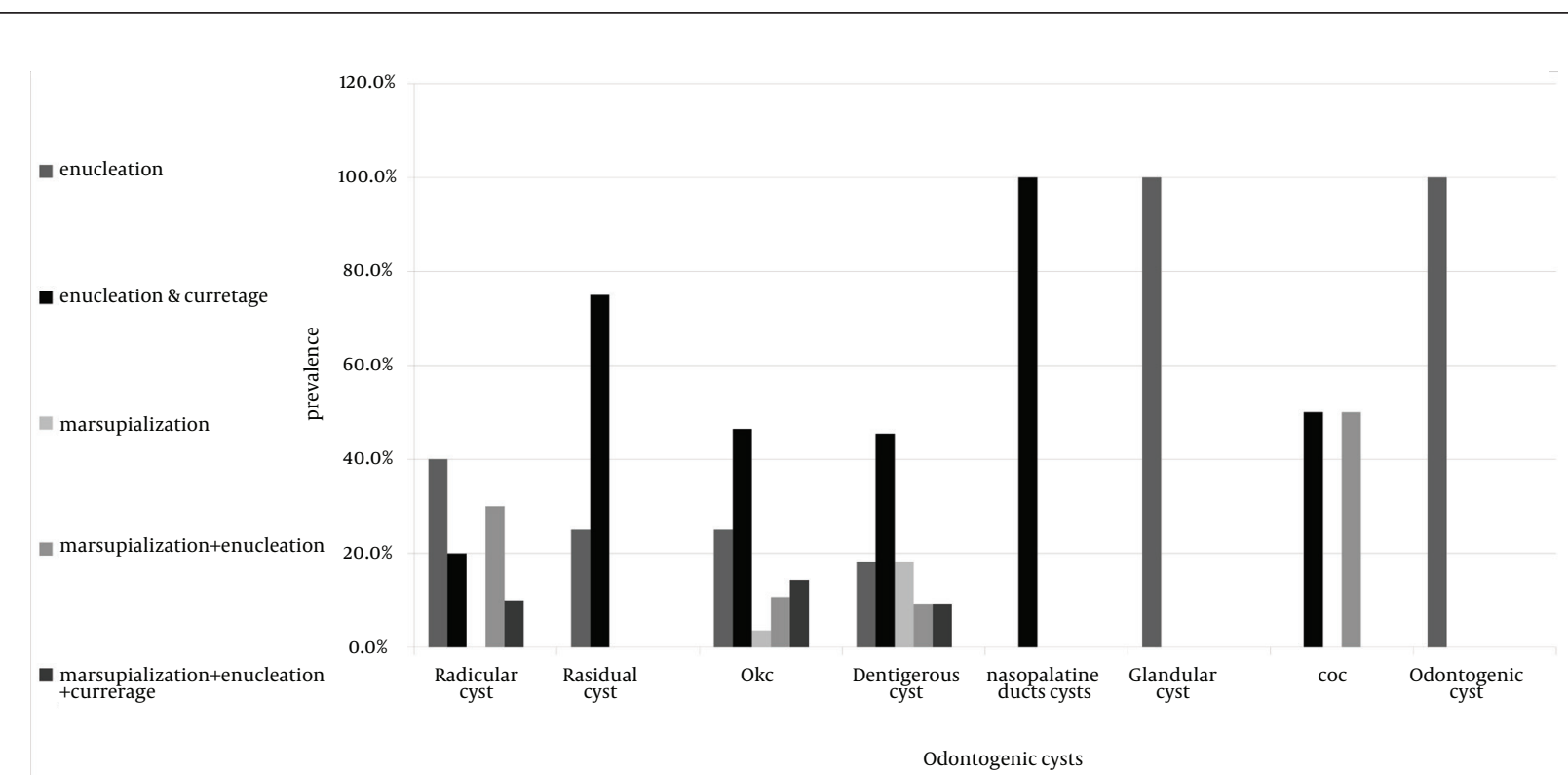

COC, calcifying odontogenic cyst; OKC, odontogenic keratocyst. 


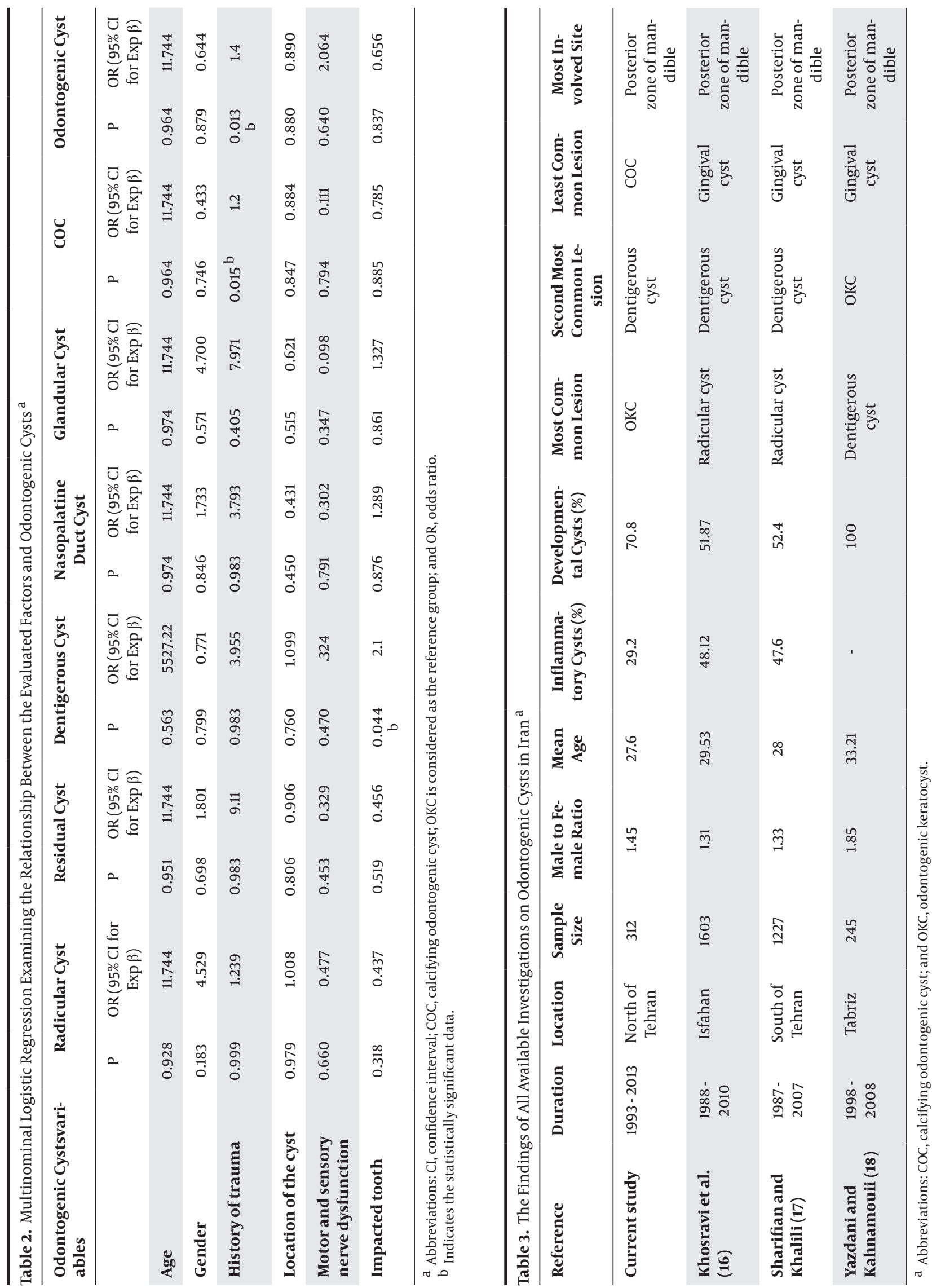




\section{Discussion}

Epidemiologic studies are helpful to understand the most common causes of diseases, frequency of lesions and the best treatments in medicine. Odontogenic cysts are very common among pathologic lesions of head and neck area. They may lead to serious problems despite their benign nature (1). Although the prevalence of odontogenic cysts are mentioned in the pathology textbooks, they are different in each population. The frequency of odontogenic cysts are studied in different investigations in various populations (19-21). Therefore, the current study aimed to investigate the factors associated with odontogenic cysts in Iranian population in the past twenty years. The age and gender distribution, trauma and positive aspiration were some of the important associated factors that the study tried to represent.

The current study selected 312 subjects referring to the hospital with an odontogenic cyst in their jaws. The cysts were categorized based on the 2005 WHO classification for odontogenic cysts and tumors. The only exception in this study was the keratocysts, which according to the WHO classification should be considered as Keratocystic Odontogenic Tumor (KOT). The reason to ignore this re-classification was the period of the survey. The study assessed the records of the past twenty years and before July 2005 the data were established as OKC. The current study compared the results of three other surveys in the Iranian population. The OKC was included in the recorded data of these studies, hence KOT was considered as an odontogenic cyst to analyze the findings of these researches. And the last criterion to include KOT in the classification was the nature of this lesion. KOT is a benign slow growing lesion with cystic behaviors. The reclassification and new molecular findings do not have any clinical implications in terms of treatment planning (22).

In the current study, OKC was the most common lesion and glandular cyst was the least common of all, although in most studies radicular cyst was the most common odontogenic cyst (23). Although in most of the investigations dentigerous cyst is the most frequent developmental odontogenic cyst (24), the current study found it the second most common lesion. These findings are considered regarding the admitted patients to the hospital. Taleghani Hospital is a governmental specialized center. It consists of 6 post specialty and 10 specialty wards. The maxillofacial section has 18 beds. Taleghani Hospital is a referral center for pathologic lesions in the city. It means that despite the number of referred patients to this center, cases with smaller cysts which can be treated under local anesthesia would not be admitted to this hospital. In similar studies performed on the Iranian population (16-18) the radicular cyst was the most common odontogenic cyst whilst the dentigerous cyst was the most frequent developmental one. Based on these results, it is concluded that OKC is in the third level and is very frequent among Iranians. The invasion and expansion patterns of this lesion are different from other cysts. The recurrence rate is much higher and treatments should be a bit more aggressive. Therefore, the clinicians should be aware of the frequency and diagnostic factors which lead them to an early diagnosis.

The most common region for all the cysts was the posterior segment of mandible. These findings are similar to those of the other studies. According to the literature, most of the odontogenic cysts are found in the posterior of mandible $(17,25)$. The location of the cyst is a good diagnostic factor for clinicians to determine the possible and differential diagnoses. The location distribution presented in Table 1 could be a useful guide to give an idea of possible cysts in each zone.

In the current study the aspiration was positive in most cases. In the aspiration of odontogenic keratocysts the fluid was mostly amber colored or brownish. The positive aspiration and the fluid color are simple and useful associated factors which may lead the clinician to differentiate cystic lesions from tumors and in some cases guess the possible diagnoses; however the negative aspiration does not deny the cystic nature of a lesion. The negative aspiration may be due to the presence of the intact cortical bone. Aspirating the cysts which did not perforate the bone and invade the surrounding soft tissue is usually negative. In some other cases such as tumors with cystic changes (unicystic ameloblastoma) aspiration may be positive and should not confuse the clinicians to detect the correct diagnosis.

In three cases of the OKCs, paresthesia of inferior alveolar nerve was the chief complaint of the patients. In all these three cases the cysts were infected. These findings shows that delayed diagnosis of odontogenic cysts may lead to important complications such as sensory nerve dysfunction. In that case early diagnosis of these lesions is important to prevent the occurrence of pathologic problems. Clinicians should be aware of the fact that although odontogenic cysts are benign they may lead to serious problems. The signs of infection in maxillofacial area with no odontogenic source should be ruled out for a pathologic lesion.

Most of the cysts had a well-defined unilocular imaging pattern. The sclerotic border can be an important sign to diagnose an odontogenic cyst in radiographic view. This finding is regarded to the slow progression of these lesions. The radiographic pattern of OKC was either unilocular or multilocular. A unilocular radiolucent lesion with well-defined and sclerotic border may be an important diagnostic factor to detect odontogenic cysts.

Age distribution is an important associated factor in onset of odontogenic cyst which was assessed in the current study. Selvamani et al. investigated 153 cases of odontogenic cysts in a south Indian sample population (9). They showed that most of the cases were in the second and third decades of their lives. The findings of the present article revealed the same results. The most common treatment plans for odontogenic cysts in the present study 
were enucleation and curettage. This treatment plan is especially important in OKC to omit the daughter cysts and prevent recurrence of the lesion. The retrospective study designed by Nunez-Urrutia et al. showed that most of the treatments performed on odontogenic cysts were enucleation and cystectomy (26), although in the current investigation most cases were cured by enucleation and curettage. The reason for this treatment plan could be the nature of odontogenic cysts. The recurrence of some odontogenic cysts is due to some small parts of the cyst called daughter cells. The recurrence of these cysts could be prevented by curettage and clearing the adjacent bone walls to reach an intact bone.

The sample size of the present study was not large enough and it may be counted as a weak point for this survey. The Taleghani Hospital is a referral hospital and covers the management of the patients in the northern part of Tehran. In order to perform a comprehensive study the data over the past two decades were assessed. Furthermore, the study designed a systematic review to present complete results of suggested issue and compensates the small sample size. The study design indicates the strong point of the investigation.

In conclusion, according to the literature the most common odontogenic cyst in the population was radicular cyst. OKC is the third most common (in the current survey it was the most common) cyst and more frequent in the second and third decades of life. The diagnosis of the odontogenic cysts would be easier by understanding the most common associated factors such as age, gender and trauma. The appropriate treatment plan is related to the nature of the odontogenic cyst. It seems that enucleation and curettage may be appropriate treatments for most of the cases.

\section{Acknowledgements}

We would like to appreciate the personnels of the hospital for their cooperation in providing the reports.

\section{Authors' Contributions}

Hassan Mohajerani was the supervisor of the project. The manuscript was written by Mohammad Esmaeelinejad. Siamak Sabour provided the data analysis. Farzad Aghdashi and Nima Dehghani helped us in collecting the data.

\section{References}

1. Slootweg PJ. Lesions of the jaws. Histopathology. 2009;54(4):401-18.

2. Regezi JA. Odontogenic cysts, odontogenic tumors, fibroosseous, and giant cell lesions of the jaws. Mod Pathol. 2002;15(3):331-41.

3. Tatli U, Erdogan O, Uguz A, Ustun Y, Sertdemir Y, Damlar I. Diagnostic concordance characteristics of oral cavity lesions. ScientificWorldJournal. 2013;2013:785929.

4. Matise JL, Beto LM, Fantasia JE, Fielding AF. Pathologic fracture of the mandible associated with simultaneous occurrence of an odontogenic keratocyst and traumatic bone cyst. J Oral Maxillofac Surg. 1987;45(1):69-71.
5. Sumer M, Bas B, Yildiz L. Inferior alveolar nerve paresthesia caused by a dentigerous cyst associated with three teeth. Med Oral Patol Oral Cir Bucal. 2007;12(5):E388-90.

6. Ochsenius G, Escobar E, Godoy L, Penafiel C. Odontogenic cysts: analysis of 2,944 cases in Chile. Med Oral Patol Oral Cir Bucal. 2007;12(2):E85-91.

7. Tortorici S, Amodio E, Massenti MF, Buzzanca ML, Burruano F, Vitale F. Prevalence and distribution of odontogenic cysts in Sicily: 1986-2005. J Oral Sci. 2008;50(1):15-8.

8. Iatrou I, Theologie-Lygidakis N, Leventis M. Intraosseous cystic lesions of the jaws in children: a retrospective analysis of 47 consecutive cases. Oral Surg Oral Med Oral Pathol Oral Radiol Endod. 2009;107(4):485-92.

9. Selvamani M, Donoghue M, Basandi PS. Analysis of 153 cases of odontogenic cysts in a South Indian sample population: a retrospective study over a decade. Braz Oral Res. 2012;26(4):330-4.

10. Acikgoz A, Uzun-Bulut E, Ozden B, Gunduz K. Prevalence and distribution of odontogenic and nonodontogenic cysts in a Turkish population. Med Oral Patol Oral Cir Bucal. 2012;17(1):e108-15.

11. Tekkesin MS, Olgac V, Aksakalli N, Alatli C. Odontogenic and nonodontogenic cysts in Istanbul: analysis of 5088 cases. Head Neck. 2012;34(6):852-5.

12. Rachanis CC, Shear M. Age-standardized incidence rates of primordial cyst (keratocyst) on the Witwatersrand. Community Dent Oral Epidemiol. 1978;6(6):296-9.

13. Shear M, Singh S. Age-standardized incidence rates of ameloblastoma and dentigerous cyst on the Witwatersrand, South Africa. Community Dent Oral Epidemiol. 1978;6(4):195-9.

14. Chavan MS, Shete A, Diwan N. Critical evaluation of the radiological and clinical features of adenomatoid odontogenic tumour. Dentomaxillofac Radiol. 2013;42(2):20120410.

15. Tanimoto K, Tomita S, Aoyama M, Furuki Y, Fujita M, Wada T. Radiographic characteristics of the calcifying odontogenic cyst. Int J Oral Maxillofac Surg. 1988;17(1):29-32.

16. Khosravi N, Razavi SM, Kowkabi M, Navabi AA. Demographic distribution of odontogenic cysts in Isfahan (Iran) over a 23-year period (1988-2010). Dent Res J (Isfahan). 2013;10(2):162-7.

17. Sharifian MJ, Khalili M. Odontogenic cysts: a retrospective study of 1227 cases in an Iranian population from 1987 to 2007. J Oral Sci. 2011;53(3):361-7.

18. Yazdani J, Kahnamouii SS. Developmental odontogenic cysts of jaws: a clinical study of 245 cases. J Dent Res Dent Clin Dent Prospects. 2009;3(2):64-6.

19. Grossmann SM, Machado VC, Xavier GM, Moura MD, Gomez RS, Aguiar MC, et al. Demographic profile of odontogenic and selected nonodontogenic cysts in a Brazilian population. Oral Surg Oral Med Oral Pathol Oral Radiol Endod. 2007;104(6):e35-41.

20. Ledesma-Montes C, Hernandez-Guerrero JC, Garces-Ortiz M. Clinico-pathologic study of odontogenic cysts in a Mexican sample population. Arch Med Res. 2000;31(4):373-6.

21. Mosqueda-Taylor A, Irigoyen-Camacho ME, Diaz-Franco MA, Torres-Tejero MA. Odontogenic cysts. Analysis of 856 cases. Med Oral. 2002;7(2):89-96.

22. Reichart PA, Philipsen HP, Sciubba JJ. [The new WHO classification of tumors of the head and neck. What has changed?].Mund Kiefer Gesichtschir. 2006;10(1):1-2.

23. Varinauskas V, Gervickas A, Kavoliūniene O. Analysis of odontogenic cysts of the jaws. Medicina (Kaunas, Lithuania). 2005;42(3):201-7.

24. Bataineh $\mathrm{AB}$, Rawashdeh MA, Al Qudah MA. The prevalence of inflammatory and developmental odontogenic cysts in a Jordanian population: a clinicopathologic study. Quintessence Int. 2004;35(10):815-9.

25. Chirapathomsakul D, Sastravaha P, Jansisyanont P. A review of odontogenic keratocysts and the behavior of recurrences. Oral Surg Oral Med Oral Pathol Oral Radiol Endod. 2006;101(1):5-9.

26. Nunez-Urrutia S, Figueiredo R, Gay-Escoda C. Retrospective clinicopathological study of 418 odontogenic cysts. Med Oral Patol Oral Cir Bucal. 2010;15(5):e767-73. 